\title{
UNA CARTA IMPRESA DEL MAESTRO ÁVILA EN UN COMPENDIO DE USO ESCOLAR DE 1554
}

\author{
POR \\ FÉlix SANTOLARIA SiERRA \\ Universidad de Barcelona
}

\section{RESUMEN}

Presentación, trascripción y edición facsímil de una carta impresa en 1554 de San Juan de Ávila. La carta, que se encuentra en un manual de formación para los Colegios de Niños de la Doctrina, se corresponde con la epístola número 169 de la edición crítica (1970) de las obras completas del Maestro Ávila.

PalabRas ClaVE: San Juan de Ávila, Gregorio de Pesquera, Juan de Lequeitio, Colegios de Niños de la Doctrina, Doctrinos, Epistolario España, Siglo XVI.

\begin{abstract}
Presentation, transcription and facsimile edition of a printed letter in 1554 of Saint John of Ávila. The letter, which is included in a manual of training for the Schools of Children of the Doctrine (Colegios de Niños de la Doctrina), corresponds with the epistle number 169 of the critical edition (1970) of the complete works of Maestro Ávila.
\end{abstract}

KEY WORDS: Saint John of Ávila, Gregorio de Pesquera, Juan de Lequeitio, Colegios de Niños de la Doctrina (Schools of Children of the Doctrine). Doctrinos, Epistolary, Spain. 16th Century.

Recibido/Received 16-04-2007

Aceptado/Accepted 16-06-2007

El sentido de esta nota es servir de breve introducción a la presentación de una carta impresa de San Juan de Ávila en 1554, recogida en un amplio com- 
pendio pensado para uso escolar, que preparó Gregorio de Pesquera, y que vio la luz en las prensas vallisoletanas de Sebastián Martínez ese año ${ }^{1}$.

Aunque se podían encontrar referencias a la obra de Pesquera en diversos repertorios bibliográficos, hasta el 2001 no se logró localizar en la Biblioteca Nacional de Lisboa el único ejemplar conocido hasta el momento ${ }^{2}$. Y desde entonces han aparecido ya varios trabajos sobre aspectos concretos de algunos de los diferentes «libros» que comprende la obra ${ }^{3}$.

Ha sido al ir estudiando la segunda parte de esta compilación (del f. $57 \mathrm{r}$ al $125 \mathrm{v}$ ), formada por varios tratados espirituales y textos diversos, recopilados por Pesquera con la finalidad doble de que sirvieran para el ejercicio del aprendizaje de la lectura y para la formación moral y cristiana de los niños doctrinos, donde ha aparecido una carta anónima y sin referencias nominales con el título de «Esta epistola escriuio vn deuoto varon amigo de Dios a otro que entendia en obras de caridad, que le pidio le escriuisse como se deuia regir para agradar a Dios en ellas» (f. 120v-121v). La carta se corresponde con la epístola número 169 del Maestro Ávila, en el volumen de sus Obras Completas dedicado al Epistolario, donde se indica que ya desde la cuarta edición ampliada del mismo, realizada en Madrid en 1595 por Juan Díaz, discípulo directo de Juan de Ávila, venía siendo recogida (f. 320v-321r)

La primera edición del Epistolario Espiritual para todos los estados de san Juan de Ávila fue hecha en 1578, nueve años después de su muerte, aunque parece ser que hubo una posible edición anterior, tal vez realizada en Baeza, de la que no se conoce ejemplar alguno, y cuya única referencia es una nota que el

\footnotetext{
${ }^{1}$ [Gregorio de Pesquera] Doctrina chrilstiana, y Espejo de bien biuir: diuid I do en tres partes. La primera es vn I dialogo o coloquio entre dos niños I con muchas cosas dela fe prouecho I sas, y la doctrina declarada y luego I la llana. En la segunda se contienen I muchas obras breues y de buena y sa I ua doctrina. La tercera tiene mu I chas coplas y cantares deuo I tos para se holgar y can I tar los niños. [Valladolid, en casa de Sebastián Martínez, 1 de mayo de 1554]

${ }^{2}$ La referencia bibliográfica primera y más completa se encuentra en J. M. SÁNCHEZ, Intento bibliográfico de la Doctrina Cristiana del P. Jerónimo de Ripalda, Madrid, 1908, (Separata de Cultura Española), 52 págs. Ver esp. 14-16. El hallazgo del ejemplar en María Emilia Balio LAVouRA (coord.), Tipografia Espanhola do século XVI. A Colecção da Biblioteca Nacional, Lisboa, Biblioteca Nacional, 2001.

${ }^{3}$ Ver, por ejemplo, Ana Martínez PereiRa, «La Doctrina Cristiana de Gregorio de Pesquera (Valladolid, 1554)», Pliegos de Bibliofilia, 17 (2002), 3-11; Mª Jesús Framiñán de Miguel, «La Doctrina cristiana de Gregorio de Pesquera (Valladolid, 1554): esbozo de análisis y contextualización históricoliteraria», Criticón, 96 (2006), 5-46. Una trascripción integra de la primera parte de la obra de Pesquera puede verse en «Una edición no conocida de la «Doctrina Cristiana» de San Juan de Ávila, incluida en la compilación de Gregorio de Pesquera: «Doctrina Cristiana y Espejo de bien vivir» (Valladolid, 1 de mayo de 1554)», Hispania Sacra, 116 (2005), 491-558.

${ }^{4}$ San Juan de Ávila, Obras Completas, Tomo V, 598-599 (edic. crítica de L. Sala Balust y F. Martín Hernández, B. A. C. Madrid, 1970).
}

Hispania Sacra, LX

121, enero-junio 2008, 173-180, ISSN: 0018-215-X 
inquisidor Alonso de la Fuente apuntó en un memorial sobre el grupo de los alumbrados de Extremadura 5 .

La fecha de esa posible edición es totalmente desconocida, pero, presumiblemente, habría de ser después de la muerte del santo. Si bien, copias manuscritas de fragmentos de sus cartas y apuntes tomados de sus homilías y catequesis circulaban libremente mientras vivía. Todo esto permite sugerir, por lo poco habitual, el posible carácter singular y curioso del hallazgo de una de sus cartas «impresa» en 1554, cuando todavía el Maestro Ávila se encontraba en plena actividad apostóli$\mathrm{ca}$, antes de su retiro a Montilla. Impresa, además, en un libro pensado como manual para los Colegios de Niños de la Doctrina. Una red de centros que se había ido extendiendo por todo el territorio de la península ibérica desde, al menos, 1542 , creados para recoger y educar a la infancia más desfavorecida de cada lugar, y que se convirtió en un amplio movimiento pretridentino de educación popular6.

¿Quién pudo ser el destinatario de la epístola? Pues, en realidad, nada podemos afirmar con certeza. No obstante, el hecho de que aparezca en un compendio para uso de los doctrinos, que es una verdadera miscelánea de textos, muchos de ellos directamente relacionados con esas instituciones (doctrina que se enseña, régimen de vida, memorial de Carlos V para que sean favorecidos, coplas y oraciones cotidianas que se hacen en los colegios, etc.), podría sugerir que tal vez también la carta pudiera tener alguna relación con alguien del movimiento de los «doctrinos».

Los fundadores oficiales de estos centros, tal como aparecen en los documentos conocidos, fueron Gregorio de Pesquera y Juan de Lequeitio 7 . De este último sabemos que fue discípulo de Juan de Ávila, y se conservan dos cartas de consejos espirituales que el maestro le escribe, por lo que tal vez pudiera ser también el destinatario de esta epístola que nos ocupa ${ }^{8}$. Hay otro dato curioso que podría resultar coincidente. El resto de los textos que contiene la segunda parte del compendio está compuesto, en general, de obras conocidas, usuales e

5 «...En unas cartas que se llaman de Ávila, impresa en Baeza,...» en Alvaro HuERGA, Historia de los alumbrados: 1570-1630, Madrid, Fundación Universitaria Española, 1978-1994, 5 vols. (Ver en el apéndice del primer volumen la trascripción y edición: Alonso de la Fuente, Nueve memoriales para el Consejo de la Santa y General Inquisición sobre los alumbrados de Extremadura. Págs. 378-442, texto cit. en 430). Manuscrito original en Archivo Histórico Nacional (Madrid), Inquisición, leg. 4443, Exp. $\mathrm{n}^{\circ} 24$, f. 32. (Cit. también en L. SAla y F. MARTín, Introducción al Epistolario, o. c., 13 n. 34, y en A. Huerga, «Conversos, alumbrados y tomismo en la «escuela» de Juan de Ávila», Teología Espiritual, 18 (1974), 229-252, esp. 232 n. 11).

6 Ver «Una edición no conocida de la «Doctrina Cristiana» de San Juan de Ávila,...» o. c., esp. 495-500.

${ }^{7}$ Fuentes documentales trascritas y editadas en «Los Colegios de Doctrinos o de Niños de la Doctrina Cristiana. Nuevos datos y fuentes documentales para su estudio», Hispania, 192 (1996), 267-290, esp. 285-287.

${ }^{8}$ San Juan de Ávila, Obras Completas, Tomo V, cartas n. 199 y 203, (edic. crítica de L. Sala Balust y F. Martín Hernández, B. A. C. Madrid, 1970). 
identificables dentro del mundo de la literatura espiritual de la época, siendo especialmente la carta la que aparece como enigmática.

Al final de todo el compendio, en el reverso en blanco del último folio (sin numerar), aparece en la parte superior una anotación manuscrita: Lequeyto. No es, desde luego, una firma. Todo parece indicar que es un apunte escrito por un lector, ¿por qué no pensar que se refiere al destinatario de ese texto incógnito de la segunda parte? Por lo demás, tanto el título como el contenido de la carta se pueden ajustar perfectamente a la tarea que estaba realizando Juan de Lequeitio: ocupado en «pobres» y obras de «caridad».

Más allá de esta cuestión, el hecho de que aparezca una carta impresa de san Juan de Ávila en el manual de Pesquera, se convierte por sí mismo en un testimonio más sobre la relación que hubo de existir entre el Maestro Ávila y su «escuela» avalista con el amplio movimiento de educación popular que representaron los Colegios de Doctrinos en las coronas española y portuguesa del mil quinientos ${ }^{9}$.

Ofrecemos a continuación la trascripción del texto y la carta en formato facsímil. En la trascripción hemos procurado modernizar la ortografía y los signos de puntuación, procurando tener en cuenta las cadencias de las frases. Hay, no obstante, algunas palabras que no hemos modificado, porque el cambio ortográfico supondría no respetar la fonética del XVI, además de que se mantienen también así, tal vez por la razón anterior, en la edición crítica (1970) del Epistolario de san Juan de Ávila.

Las variaciones más destacadas entre las dos ediciones de la carta (1554 y 1595 [1970]) corresponden al título y al tratamiento que se da al destinatario, más próximo en Pesquera (hermano, vos), y más formal en la edición de 1595 (vuestra merced). Tanto el título como el tratamiento solían ser los aspectos que habitualmente se prestaban a ser más modificados en aquellos momentos por los editores de este tipo de epístolas espirituales. El primero porque suele ser siempre creación del editor y no del autor de las cartas, y el segundo porque, por respeto a la intimidad del destinatario o de sus familiares, especialmente en las cartas editadas en el propio siglo XVI, por proximidad temporal, se procura el anonimato eliminando nombres y datos (personas, lugares y fechas), sustituyéndose por un tratamiento genérico. (Fol 120v)

«Esta epístola escribió un devoto varón amigo de Dios a otro que entendía en obras de caridad, que le pidió que le escribiese como se debía regir para agradar a Dios en ellas.

\footnotetext{
${ }^{9}$ Una síntesis de las posibles relaciones de Ávila con la red de colegios doctrinos en «Una edición no conocida de la «Doctrina Cristiana» de San Juan de Ávila, incluida en la compilación de Gregorio de Pesquera: «Doctrina Cristiana y Espejo de bien vivir» (Valladolid, 1 de mayo de 1554)», Hispania Sacra, 57 (2005), 491-558.
}

Hispania Sacra, LX

121, enero-junio 2008, 173-180, ISSN: 0018-215-X 
Muy amado hermano en Jesucristo, días ha que recibí una carta vuestra por la cual por la cual vi la merced que nuestro Señor os ha hecho en tomaros por instrumento y causa segunda para hacer misericordia con vuestros prójimos, o por mejor decir, con El mismo, pues es tanto su amor con ellos, que tomó por hecho a El lo que a ellos se hace.

Sabed hermano agradecer (/Fol 121r) esta merced y conoceros por indigno de ella, pues no es a todos dado del todo emplearse en las obras de nuestro Señor, e gozaos de la merced mirando que Dios quiere recibir de vos servicio por tener ocasión de galardonaros. Temed vuestra flaqueza y la alteza de la obra, no seáis hallado falto en ella. Acordaos de la diligencia que ponen los hombres en los negocios que sus señores les encomiendan, cuanto más si tocan a los mismos señores en sus personas, e vivid con cuidado, mirando que os ha encomendado nuestro Señor una cosa por cuyo amor puso El la vida.

Llamadle pidiéndole gracia, y esperadla, pues os dio la primera, y traed siempre delante vuestros ojos al mismo Señor puesto en cruz e cercado de nuestros dolores, e por El esforzaos a sufrir pesadumbres de pobres, y a saber llevar a cada uno como es menester, haciéndoos todo a todos para que aprovechéis a todos.

Y entre estas cosas no olvidéis vuestra propria ánima de darle el cebo que a menester para estar fuerte, porque algunos hay que so color de aprovechar a otros, dejan sus ánimos sin oración, sin lección e sin semejantes ejercicios, e así faltan en la obra exterior o la hacen mal hecha, porque faltando el interior esfuerzo, todo va lleno de flaquezas, e para tener éste es menester ganarlo de nuevo, que de otra manera no hay caudal, por grande que sea, que no se gaste si sacan de él y no ganan.

Y por eso es comparada la oración, con mucha razón, al sueño, porque en ella cobra el ánima nuevos espíritus, como el cuerpo en el sueño, y la palabra de Dios (/Fol 121v) al manjar, porque restaura lo que con las ocupaciones, aunque buenas, perdemos.

El camino es estrecho, mirad no os apartéis a una parte o a otra, o entendiendo en vos sólo, o en los otros con olvido vuestro.

Haced como el Señor, que velaba y oraba hasta sudar sangre, luego visitaba y consolaba a sus discípulos, y después tornaba a la oración.

Para darnos doctrina que se ayudan maravillosamente tratar con Dios y hacer bien a prójimos. Y que lo uno sin lo otro es seco y estéril.

El Señor os enseñará y allá tenéis personas con quien podáis tomar consejo sobre la orden que debéis tener en particular sobre este negocio.

Cristo, que lo comenzó, lo acabe para su gloria. Amén. 


\section{tibro.}

Suple:loefecto s nueftro amoz teniedo nofocros pue fto enel toda nuefra confiança. If payozes fuerô las afliciones $z$ paffion que cbitito padescio que la pena que todos nueftros peccados auian merecido ni me: receranjamas. C. Todas las vejes que offendemos a cissifo le crucificamos/ze eftas maneras o crucificar a cbsifto las oeuemos oe tener po: muscho masterria bles a crueles y mas deuemos oe como vernos a lagri mas con verdadera trifteja de cozacon/ pu:que como aquella pzimera vesä cbzifto fue crucificado fue para nuefra lalud/anfi eftotras fon nueftra muerte oe lag quales nos conuleue guardar mucbo. C 2Bien atuen turados los que mueren enel iefio.

TExnzioz rs el-oia oela maerte que el oia oél narcis miento.

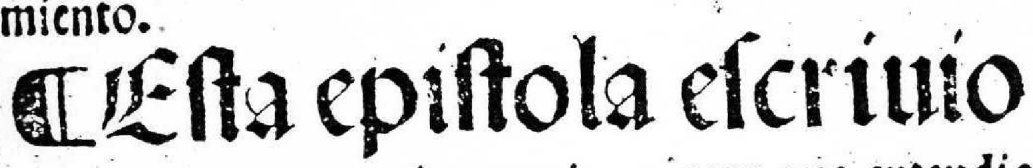

viloeuoto paronamigo de diog a otro que entendia en obzas oe caridad que le pidio le efcriaiffe como fes uia regir para agradar a oios enellas.

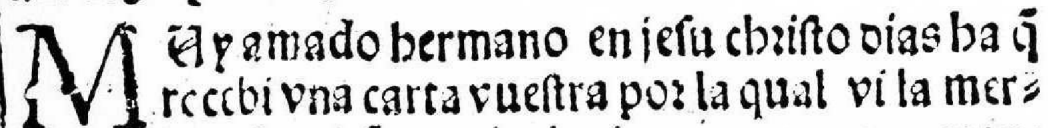
cid que nueftro fentio: os babeciso en tomaros poz in ftrumento y caula fegunda para bajer mifericosdia con vueit ros azorimos/o poz mejoz oejir conicl nifino pueses tanto fa amos conellos que tomo pos becbo a dl o que a ellos febase. Sabed bermano ayradefecr 
$\checkmark$

Segundo.

So.crí.

etta merced p conoceros poz indigno oella pues no es a todos oado oel todo emplearfe en las obzas oe niro ferios.z 50590 sela merced mirando que oios quiere recebir oe vos feruicio poz tener ocafion s galardona

- ros:temed vueftra flaqueja y la alteja oela obza/no feays balladofalto enella-acosdaos oela oiligencia à poné los bombzes en 103 negocios que fus fetiozesles encomiendan quanto mas fit tocan alos mifmos fefic res en fus perfonas / $z$ biuid con cupdado mirando ä os a encomendado nueftro ferio: vna cofa pos cugos mo: puro el la vida. Llamadle pidiendole gracía 9 \& perad la pures os oio la pzimera y traed fiemp:e $\checkmark$ lăt bị̂os ójus al mírmoréíoz puefto en crus z sercado o nuefros oolozes/z posel effozços a fuirtir peiadur b:es oe pobzes/ya faber lletuar a cada vno como eim nefter bajiendo os todo a todos para que ayzouecls ysa todos: pentic eftas cofas nooluiders vueftra. pia anima se oarle el celo que a minefter para cf? fuerte: pezque algunos ay que fo coloz oe apzouedb: a otros ocran fus animos in ozacion fin lecion $z$ fin meiantes erercicios/zaffi faltan enla obza ericrioz 0 bajen mal becba pozque fultando el interioz eifuer todo valleno oe flaquejas z para tener efte es mene ter ganarlo se nue uo /ique oe otra manera no af ca dal poz grande que fea şue no le gafte fi facan sil ? sanan. $Y$ pozeffo es comparada la ozacion con $m$ cba razó al freeío pozị enella cobza el anima nueu épiritus como el cuerpo enill fueño y la palabza ò 


\section{tibro.}

artianar:posque reftaura lo que con las ocupacionei autit buenas perdenos cl camino es efrectoo mirad no os aparteys amia parte os otra $/ 0$ entendiendoen vos foto o en los otros con oludido vio. Foned como elfentos qac velana y ozaua baita fudar fangte/ lues so vilitats $y$ confolatua a fus oifcipulos $/ Y$ sipues $t$ o

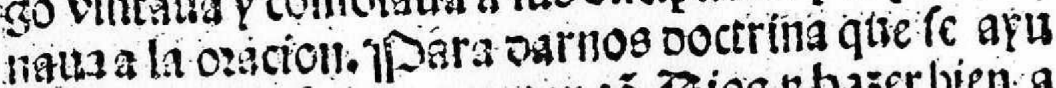
dan marauillofantente trater có Bzios y bajer bien a ptoximoa:yqu lo vno fin lo otro es feco y efteril. Letres tio2 os enfefira y alfa teriçs pertonas con quien po days tomar colliéto fobze la bo:den que ocueys tener en partieular fouze encenegocio. Elviffo quelo come colosabe parafu g!osia. 2Amen. Uefro queleligue fepnedeoe. jir guando alguno eftudere con algun enfers mo que efta ala muerte/omuriendole.

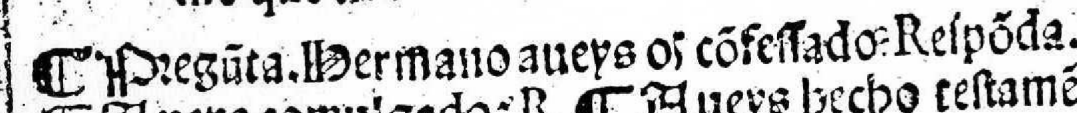

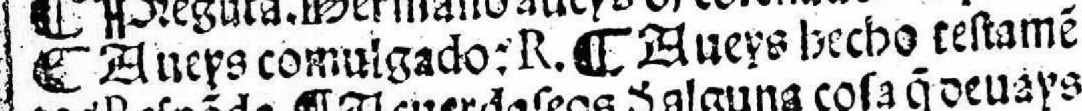
to:Refpóda. TI Tu cuerdareos Salguna cora goeuays a alsund $/ 0$ feaps encarzo\%"Refonda. Al cuerdafeor

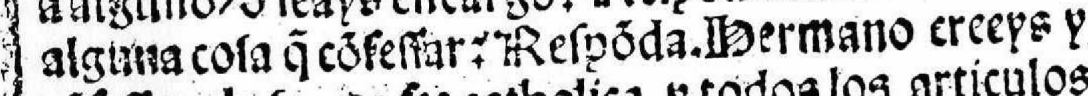
cóferfaygla fancta fece catbolica y todos los articulos oella/anfl comola fancta madre pglefia? R. Si creo. TIPsoterays oe mosir en ella como catbolico a fic

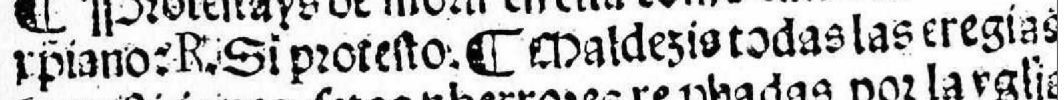
fupueficiones/fetas yberrozes repbadas poz la rgli:

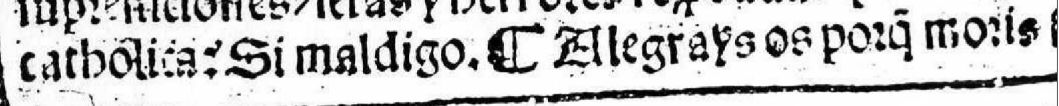

